\title{
Registries for orphan drugs: generating evidence or marketing tools?
}

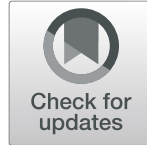

\author{
Carla E. M. Hollak ${ }^{1,2^{*}}$ (D), Sandra Sirrs ${ }^{3}$, Sibren van den Berg ${ }^{2}$, Vincent van der Wel ${ }^{2}$, Mirjam Langeveld ${ }^{1}$, \\ Hanka Dekker ${ }^{4}$, Robin Lachmann ${ }^{5}$ and Saco J. de Visser ${ }^{2}$
}

\begin{abstract}
Independent disease registries for pre-and post-approval of novel treatments for rare diseases are increasingly important for healthcare professionals, patients, regulators and the pharmaceutical industry. Current registries for rare diseases to evaluate orphan drugs are mainly set up and owned by the pharmaceutical industry which leads to unacceptable conflicts of interest. To ensure independence from commercial interests, disease registries should be set up and maintained by healthcare professionals and patients. Public funding should be directed towards an early establishment of international registries for orphan diseases, ideally well before novel treatments are introduced. Regulatory bodies should insist on the use of data from independent disease registries rather than company driven, drug-oriented registries.
\end{abstract}

Keywords: Registries, Orphan drugs, Real world data, Disease registries

\section{Orphans}

Rare diseases (defined in the EU as affecting fewer than 1 in 2000 people) are individually rare but collectively common, affecting $6-8 \%$ of the population [1]. Orphan drugs, drugs for rare diseases, are developed under specific regulations in the EU, the United States and Canada with incentives to stimulate pharmaceutical companies to develop medicines for rare diseases. The idea is that investments in orphans would not be commercially attractive. However, several studies suggest that orphan drugs are associated with a higher return on investment than drugs licensed for common diseases resulting in intense pharmaceutical industry interest in rare diseases $[2,3]$. While the number of treatable rare conditions is still relatively low, the orphan drug market is expanding

\footnotetext{
*Correspondence: c.e.hollak@amsterdamumc.nl

'Department of Endocrinology and Metabolism, Amsterdam University Medical Centers, location Academic Medical Center, University of

Amsterdam, F5-170, P.O. Box 22660, 1100, DD, Amsterdam, The Netherlands 2Platform Medicine for Society at Amsterdam University Medical Centers, location Academic Medical Center, University of Amsterdam, Amsterdam, The Netherlands

Full list of author information is available at the end of the article
}

at an annual growth rate of $11.2 \%$ and orphan drugs are expected to make up more than $18 \%$ of worldwide prescription drug costs by 2024. [4]. The majority are for oncology indications followed by cell- and gene-based therapeutics, the latter typically indicated for ultra-rare inherited diseases [5].

\section{The development process and registries}

Orphan designation is granted to an applicant at the beginning of the drug development process based on the "medical plausibility" of the proposed active substance's effect on a rare disease. However, conducting clinical studies to prove safety and effectiveness can be challenging due to small patient numbers and phenotypic variability. Because they are developed for rare diseases, orphan drugs may receive marketing authorization with limited safety and efficacy data [6]. Societal pressure for early access to these novel therapies is high. Orphan drug registration and authorisation is organized by authorities such as the Food and Drug Administration (FDA) in the US, Health Canada in Canada and the European Medicines Agency (EMA) in the EU. Data 
from disease registries can play a role in this process, helping to describe the natural history of the disease and identifying suitable clinical and surrogate endpoints for clinical trials [7].

\section{Post-registration and registries}

Registries also have a role after marketing authorisation when regulatory authorities request data on the realworld effectiveness and/or safety profile of new drugs. Currently, the notion that real world data can be used in regulatory decision making is increasing [8]. Since clinical trials in rare diseases may be underpowered and of short duration, long-term data are needed to determine the optimal place of these treatments in disease management. Post-registration studies can be used to support recommendations in treatment guidelines. For instance, in the EU, the EMA may grant a conditional marketing authorisation, when the data provided in a marketing application is less comprehensive than would be accepted for a non-orphan indication [9]. The applicant is then obliged to provide comprehensive data to confirm the positive risk-benefit ratio of their product in commercial use but, when it is unlikely such data will be available, the EMA may still grant a marketing authorisation under exceptional circumstances. In these situations, a post-marketing registry may be required by the regulatory authorities or proposed by the applicant as part of the risk management plan.

\section{Real world data and industry}

The development of registries to collect real world and life data for orphan drugs is largely driven by the requirements imposed by regulators on applicants for marketing authorisation. These requirements do not always result in the development of registries with open access to real-world data. Firstly, many of these registries (which are drug focused rather than disease-oriented) have been set up to fulfil marketing obligations. Engagement of patient representatives and healthcare professionals is very important to help identify the most clinically relevant outcome parameters and indicators of quality of life [7]. However, this input comes generally too late and is therefore insufficient to capture adequate information for biomarker research, natural history and optimal disease management, which will be necessary for evaluation of effectiveness and appropriate use. Secondly, regulatory agencies can request that data are collected following Good Clinical Practice standards, however, they are frequently unable to review whether these data are sufficiently consistent and relevant. Data sets may be incomplete making it impossible to distinguish subgroups of patients with different phenotypes and differential risk of suffering defined disease complications [10]. Thirdly, the operation of these industry- sponsored registries may result in conflicts of interests between pharmaceutical companies, healthcare professionals and patients. For instance, data from these registries can only be accessed and published with the support of the sponsor. These publications, based on data owned by the industrial sponsor, are then often used as the basis of clinical guidelines. Meetings of clinical experts are convened by the sponsor to draft guidelines (often facilitated by industry-hired medical writers) which depend heavily on expert opinion and systematic literature reviews of publications which are mostly based on registry data [11]. Because the data from industry funded registries may not be freely exchangeable or accessible for analysis by third parties, there is a risk that publications from registries, and the guidelines that arise from them, may be biased [12].

\section{Orphan drugs for non-oncology indications}

Orphan drugs for rare (non-oncology) indications account for 88 of the 129 currently authorized orphan drugs [13] and, as is typical in rare diseases, no other specific treatments were available at time of initial marketing authorisation. In almost $30 \%$ of orphan drug approvals (Table 1), the orphan drug was authorised by EMA either with conditional approval or approval under exceptional circumstances, usually resulting in the setup of a registry. In addition, in half of the 'regularly' approved cases a registry was launched. In total, $62,5 \%$ of approvals coincided with registry establishment.

\section{Registries and industry}

The majority of the registries requested or suggested in the approval process of orphan drugs (41 of 55) have been initiated and funded by industry (Fig. 1). In only 5 cases non-industry registries were set up for regulatory approval (cystic fibrosis for two products, spinal muscular atrophy, Cushing Syndrome, and Hemophilia B). For 4 drugs, an industry-initiated registry not mandated for regulatory purposes was established. Almost all these industry registries have a set reporting date and it is not clear what will happen to the registry after that date.

\section{Lysosomal storage disorders}

Over the last 25 years a number of new drugs have been licensed for use in lysosomal storage disorders (LSD's). Many of these have associated post-marketing registries to address long-term safety and effectiveness outcomes. These registries provide an example of how these regulatory requirements are set up and used in practice (Table 2).

LSD's are a group of ultra-rare disorders with a prevalence ranging from less than 1 in 100.000 up to 1 in 30.000 [16]. These disorders are inborn errors of metabolism caused by a deficient enzyme, with in general a 
Table 1 Approval types and registries used for post-marketing regulatory purposes for orphan (non-oncology) drugs from 2000 to 2019. (data from the EU PAS register [14], European public assessment reports (EPARs) [13, 15] or publicly available information)

\begin{tabular}{llll}
\hline Approval type & Subtotal & Registry not part of approval & Registry part of approval \\
\hline Conditional approval & 9 & $1(11,1 \%)$ & $8(88,9 \%)$ \\
Exceptional circumstances & 16 & $0(0 \%)$ & $16(100 \%)$ \\
No conditional approval or exceptional circumstances & 63 & $32(50,8 \%)$ & $31(49,2 \%)$ \\
Total & $\mathbf{8 8}$ & $\mathbf{3 3 ( 3 7 , 5 \% )}$ & $\mathbf{5 5}(\mathbf{6 2 , 5 \% )}$ \\
\hline
\end{tabular}

wide array of phenotypes. The prototype treatment is enzyme replacement therapy (ERT): intravenous administration of a recombinant enzyme, which supplements the deficiency to degrade stored macromolecules.

Gaucher disease was the first LSD shown to be treatable by enzyme replacement. Treatment with Ceredase, a placental derived enzyme, resulted in reversal of massive hepatosplenomegaly and cytopenias and prevented severe bone complications: a landmark achievement. Genzyme corporation (now a Sanofi company) entered this ultra-orphan area under the USA orphan drug act, granting them 7 years of market exclusivity. At that time Ceredase was the most expensive medicine ever: treatment of a $50 \mathrm{~kg}$ patient with the licensed dose came in at cost between 50.000 and 500.000 USD per year [17]. However, it soon became clear that, as a result of variability in phenotypes, not all patients needed treatment and those that did could often be treated with lower doses than the licensed dose [17].

\section{Enter registries}

Following debates on dosing and differences in the natural disease course, a 1995 Health Technology Assessment meeting resulted in a recommendation to seek better evidence to support the appropriate use of this costly therapy [18]. Also for this purpose, Genzyme set up a global registry in 1991 containing data on both treated and untreated patients: run and financed by Genzyme, analyses performed by Genzyme and publications supported by Genzyme. An international board of Gaucher treating physicians helped to operate the registry: meetings were held, with key opinion leaders, paid by Genzyme, discussing treatment goals and diagnostic

\section{Non-cancer orphans (88)}

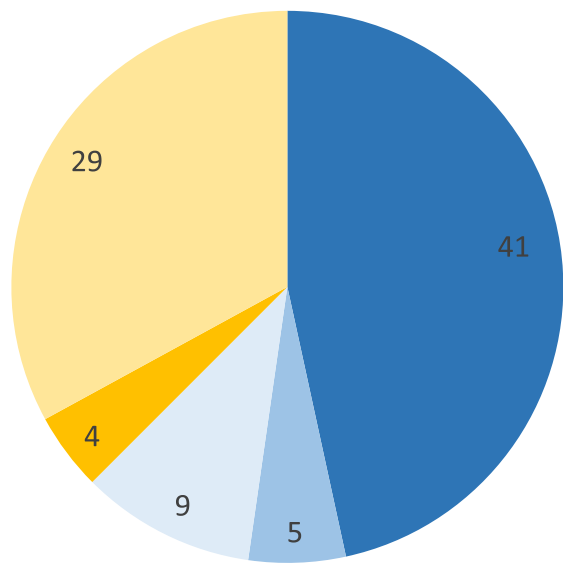

Required - Industry-registry $\quad$ Required - Non-industry-registry

Required - Not found or not recruiting $\square$ Not required - Industry-registry

Not required - None found

Fig. 1 Origin of registries (data from the Encepp PAS database, risk management plan, European public assessment report (EPAR) or publicly available information) [13-15] 
Table 2 Overview of the LSD disease registries sponsored by pharmaceutical industry

\begin{tabular}{lllll}
\hline Disease & Registry & $\begin{array}{l}\text { Year } \\
\text { established }\end{array}$ & Sponsor & $\begin{array}{l}\text { Patients enrolled } \\
\text { (N) }\end{array}$ \\
\hline LAL-D & Lysosomal Acid Lipase (LAL) Deficiency Registry (ALX-LALD-501) & 2012 & Alexion & 1000 \\
Fabry & Fabry Disease Registry & 2001 & Genzyme, a Sanofi Company & 9000 \\
& Fabry Outcome Survey (FOS) & 2001 & Shire & 4000 \\
Gaucher & International Collaborative Gaucher Group (ICGG) Gaucher Registry & 1991 & Genzyme, a Sanofi Company & 12,000 \\
& Gaucher Disease Outcome Survey (GOS) & 2010 & Shire & 1257 \\
MPS I & Mucopolysaccharidosis I (MPS I) Registry & 2003 & Genzyme, a Sanofi Company & 1500 \\
MPS II & Hunter Outcome Survey (HOS) & 2005 & Shire & 2000 \\
MPS & A Multicenter, Multinational, Observational Morquio A Registry & 2014 & BioMarin Pharmaceutical & 583 \\
IVType A & Study (MARS) & & \\
MPS VI & Mucopolysaccharidosis (MPS) VI Clinical Surveillance Program (CSP) & 2005 & BioMarin Pharmaceutical & 200 \\
MPS VII & Mucopolysaccharidosis VII Disease Monitoring Program & 2018 & Ultragenyx Pharmaceutical Inc & 35 \\
Pompe & Pompe Disease Registry & 2004 & Genzyme, a Sanofi Company & 2000 \\
& Alglucosidase Alfa Pompe Safety Sub-Registry & 2015 & Genzyme, a Sanofi Company & 110 \\
& Pompe Lactation Sub-Registry & 2012 & Genzyme, a Sanofi Company & 5 \\
& Pompe Pregnancy Sub-Registry & 2011 & Genzyme, a Sanofi Company & 20
\end{tabular}

algorithms. These discussions resulted in manuscripts, (written by a medical writer hired by Genzyme), that were published in peer reviewed journals. Interestingly, none of the Gaucher Registry publications reported on limitations of treatment with enzyme replacement therapy [19]. A similar approach for Genzyme's (now Sanofi) oral drug for Gaucher (Eliglustat) resulted in a treatment algorithm publication, without mentioning the possibility of the patient having mild disease status that does not require treatment [20].

\section{New drug, new registry}

When their competitor Shire (now Takeda) launched a similar ERT in the EU in 2014, another global postmarketing registry for Gaucher disease was started (initiated in 2010) to address the same issues of unmet medical needs, diagnostic challenges and treatment goals. Both companies financially supported separate sponsored symposia, round tables for educational purposes and investigator-initiated studies. These frequently led to recommendations for early initiation of therapy or population screening, including newborn screening (NBS) to find new patients. A pilot NBS study on NBS for a panel of lysosomal storage disorders did not identify any early onset phenotypes which might have benefited from presymptomatic initiation of therapy [21].

\section{Fabry disease}

Following the success of ERT in Gaucher disease, the second disorder that was targeted was Fabry disease, a disease that can cause neurologic, renal and cardiac dysfunction. In this case, Genzyme and Shire were both able to market their enzymes at the same time in the $\mathrm{EU}$ at a mean similar price of EUR 200.000 per patient per year. Two separate global registries (initiated in 2001) were mandated by the EMA to address open questions concerning effectiveness and safety. There has been no exchange of data or collaborative analysis of data from the two registries. Separate, sponsored groups have addressed the same questions following the pattern described for Gaucher disease. Unfortunately, ERT for Fabry disease has proven to be less effective in preventing complications than ERT for Gaucher disease. The diversity of phenotypes, lack of knowledge about differences in the natural disease course in the different patient groups and the development of treatment interfering neutralizing antibodies in many patients has hampered our ability to draw any robust conclusions [22]. There remains real uncertainty about whom to treat, at what dose and for how long. It is clear that the registries were not set up to be able to answer these questions, since crucial data e.g. on patients' disease phenotype, were usually missing and results from the different antibody assays used by the companies could not be compared [23].

\section{Independent registry for Fabry disease}

These uncertainties have led to some independent initiatives, including the Canadian Fabry Disease Initiative and a European database, that eventually led to the generation of some independent guidelines and recommendations for diagnosis and treatment start and stop 
criteria [23, 24]. However, the long-term uncertainty about the effectiveness of treatment has impacted reimbursement decisions: in the Netherlands, in 2011, a provisional decision to stop reimbursement of both ERT's for Fabry, and ERT for Pompe disease, based upon the poor quality of data, led to national uproar. Not only was the quality of the data available insufficient to make informed decisions, but the existence of the EMA-mandated post-marketing registries hampered the set-up of independent databases: healthcare professionals were not keen to contribute to yet another database for which there was no financial support for data monitoring, meetings and publications. In fact, by putting the pharmaceutical industry in charge, regulators have accepted that companies become involved in the set-up of guidelines, which carries a risk for biased recommendations $[11,12]$.

\section{Lysosomal acid lipase deficiency}

Following the Gaucher HTA meeting and call for natural history studies early in the process of drug development, companies have become increasingly interested in premarketing data acquisition. Examples of this are the initiatives for pre-marketing data collections for lysosomal acid lipase deficiency (LALD), an ultra-rare LSD with variable presentation which in its severe forms may lead to liver failure. The manufacturer of ERT for this disorder, Synageva, later acquired by Alexion, sponsored publications on untreated patients in the pre-marketing phase, pushing the message through their key opinion leaders that LALD is a devastating disorder, with a progressive course, in all patients [25]. Thus, once diagnosed, patients would need to be treated with their product, sebelipase alfa, that comes at a price of up to 800.000 Euro per patient per year. However, no longterm follow-up of untreated, mildly affected patients has ever been presented. Anecdotal experience suggests that patients at the milder end of the spectrum may not benefit from therapy at all. In addition, it is also not clear that long-term complications can be prevented by treating patients with severe or advanced disease. However, physicians seeing a single patient or family will rely on published recommendations, and end up treating their patients. It is no surprise that Alexion has set up a Global LAL Deficiency Registry in 2012 to document the use of their product.

\section{From drug- to disease registry}

The current variable approach to real-world data collection with a predominance of industry owned, drugoriented patient registries is not fit for purpose and should be reassessed. Since several stakeholders contribute to the current system, recommendations for change should be addressed to all. Firstly, there needs to be change in the mindset of healthcare professionals towards the ownership of patient data. This implies that they should be pro-active in the set-up of independent, sustainable disease registries, which are then governed by patients and healthcare professionals together [26]. These databases should contain all data needed to gain a full picture of the natural history and optimal management of disease, as well as the data needed to answer the very specific questions about safety and outcomes asked by regulators and industry. Secondly, pharmaceutical companies should accept the call for generation of post-marketing evidence for healthcare that is free of commercial influences. Recently, an international group of scientists has published pathways to independence, clearly pointing out that this is needed for trustworthy evidence [27].

Thirdly, regulatory authorities have launched several initiatives to use post-marketing evidence generation for regulatory decision-making [28]. This should not be undertaken without healthcare professionals and patients as necessary partners and should use data from independent patient registries to avoid conflicts of interest [29].

\section{Registry funding}

Lastly, governments and/or regulatory authorities should make commitments to fund accessible patient/disease registries, which will then be independent of industry [27] For rare diseases, registries should be initiated long before a novel treatment receives marketing authorisation. If there isn't a disease registry in place at the time of orphan designation for a new drug, then that would be a good time to start one. Once registries have been established, then ongoing structural funding can come from other sources, including industry, in the form of mandatory fees which could be linked to clinical trial registration and market authorisation. Such fees should not raise the costs of orphan drugs, since industry sponsored registries would no longer be needed. In addition, public funding could be made available for investigator-initiated studies e.g. on appropriate use, conducted by centres of expertise, who will maintain the registry. EMA/FDA and/or other regulatory bodies including HTA bodies making reimbursement decisions can further empower independent registries by directing post (or even pre) marketing research to these registries.

\section{Conclusion}

The undue influence on clinical practice of the use of post marketing registries as marketing tools is underestimated. The virtual absence of independent registries for rare diseases results in unwanted conflicts of interest. Health care professionals and patient organizations, operating independently of industry, should take responsibility for providing and generating independent data which can be used to (re) evaluate registration/reimbursement decisions and guide optimal patient management in the challenging field of treating patients with rare diseases. 


\section{Acknowledgements}

Not applicable.

\section{Authors' contributions}

$\mathrm{CH}$ and SdV have made the concept for the article; all authors have contributed to the content and critical review of the article. $\mathrm{CH}$, SS and ML are healthcare professionals involved for many years in treatment of rare disorders with orphan drugs in different areas in the world. SdV is a clinical pharmacologist involved in organizing and funding drug and/or disease registries in the Netherlands since 2011 and supporting the platform "Medicine for Society". SvdB is a PhD student with a master in drug innovation, who studies the usefulness of orphan drug registries. VvdW has a master degree in health economics, policy and law and is employed by the Amsterdam University Medical Centers related to the platform "Medicine for Society" and the Fair Medicine Foundation, which aims to develop novel pharmaceutical business models. HD is a patient representative, employed by the Dutch Association for Inherited Metabolic Diseases (VKS) and represents the patient perspective on development and authorization of orphan drugs for patients with inherited metabolic diseases.

$\mathrm{CH}$ and SdV are responsible for the overall content as guarantors. The corresponding author attests that all listed authors meet authorship criteria and that no others meeting the criteria have been omitted. The authors read and approved the final manuscript.

\section{Funding}

This work was supported by the "Vriendenloterij" part of Dutch Charity Lotteries, that funds the platform "Medicine for Society". Medicine for Society was launched in 2019 as an idealistic project related to the Amsterdam UMC with the aim to support accessibility and affordability of drugs for rare diseases.

\section{Availability of data and materials}

The datasets used and/or analysed during the current study are available from the corresponding author on reasonable request.

\section{Ethics approval and consent to participate}

Not applicable.

\section{Consent for publication}

Not applicable.

\section{Competing interests}

$\mathrm{CH}$ receives payments from the National Healthcare Institute (The Netherlands) as member of the "Horizonscan" for new drugs for metabolic and endocrine disorders and the Dutch Advisory Committee to the insured Package (ACP). She is an unpaid member of Takeda's Humanitarian Aid Program.

$\mathrm{CH}$ and $\mathrm{ML}$ are involved in pre-marketing studies with orphan drugs for which the Amsterdam University Medical Center receives financial compensation for study costs from Sanofi, Protalix and Idorsia.

SS participated in research projects funded by Sanofi, Takeda, Idorsia, Actelion, and Amicus and received support for travel to attend educational meetings from Amicus and Sanofi. She participated in an advisory board for Amicus. The Adult Metabolic Diseases Clinic at Vancouver General Hospital receives unrestricted grant funding from Sanofi, Takeda, and Amicus to support the services of a genetic counselor for patients of the clinic. She is an unpaid member of the multidiscliplinary committee that provides input to our provincial ministry of health on the use of drugs for rare diseases. VvdW is director and shareholder of the company "Patient One", which develops and orphan drug according to Fair Medicine principles. He is employed by the Fair Medicine Foundation, that is financed by the Dutch government to develop novel pharmaceutical business models. HD receives payments from the National Healthcare Institute as member of the "Horizonscan" for new drugs for metabolic and endocrine disorders. VKS has received financial compensation for work on patient friendly texts from Lysogene and Chiesi. She receives travel support but no fees for patient advisory boards of Sanofi, Takeda, Ultragenyx and Orchard Therapies. RL has received consulting fees from Sanofi/Genzyme, Biomarin and Kyowa Kirin International and honoraria and travel support from Sanofi/Genzyme and Takeda His department receives staff funding from Kyowa Kirin
International and research grants from Nutricia. He is unpaid Chair of the Scientific Committee of the Recordati Rare Diseases Foundation. SvdB and SdV declare no conflicts of interest.

\section{Author details}

'Department of Endocrinology and Metabolism, Amsterdam University Medical Centers, location Academic Medical Center, University of Amsterdam, F5-170, P.O. Box 22660, 1100, DD, Amsterdam, The Netherlands. ${ }^{2}$ Platform Medicine for Society at Amsterdam University Medical Centers, location Academic Medical Center, University of Amsterdam, Amsterdam, The Netherlands. ${ }^{3}$ Division of Endocrinology and Metabolism, University of British Columbia, Vancouver, British Columbia, Canada. ${ }^{4}$ KKS, The Dutch patient association for Inherited Metabolic Diseases, Zwolle, Netherlands. ${ }^{5}$ Charles Dent Metabolic Unit, National Hospital for Neurology and Neurosurgery, London, UK.

Received: 16 June 2020 Accepted: 24 August 2020

Published online: 03 September 2020

\section{References}

1. Auvin S, Irwin J, Abi-Aas P, Battersby A. The problem of rarity: estimation of prevalence in rare disease. Value Health. 2018;21:501-7.

2. Rodriguez-Monguio R, Spargo T, Seoane-Vazquez E. Ethical imperatives of timely access to orphan drugs: is possible to reconcile economic incentives and patients' health needs? Orphanet J Rare Dis. 2017;12:1.

3. Hughes DA, Poletti-Hughes J. Profitability and market value of orphan drug companies: a retrospective, propensity-matched case-control study. PLoS One. 2016;11(10):1-12

4. Pomeranz K, Siriwardna K, Davies F. Orphan drug report 2020. Report by EvaluatePharma; 2020

5. Pomeranz K. Orphan drug report 2019. Report by EvaluatePharma; 2019.

6. Schuller Y, Biegstraaten M, Hollak CEM, Klümpen HJ, Gispen-de Wied CC, Stoyanova-Beninska V. Oncologic orphan drugs approved in the EU - do clinical trial data correspond with real-world effectiveness? Orphanet J Rare Dis. 2018:13:214

7. Kodra Y, Weinbach J, Posada-de-la-Paz M, Coi A, Lemonnier SL, van Enckevort D, et al. Recommendations for improving the quality of rare disease registries. Int J Environ Res Public Health. 2018;15:1644.

8. Cave A, Kurz X, Arlett P. Real-world data for regulatory decision making: challenges and possible solutions for Europe. Clin Pharmacol Ther. 2019; 106(1):36-9.

9. Marketing authorisation [Internet]. Amsterdam: European Medicines Agency; c1995-2020 [cited 2020 Jan 14]. Available from: https://www.ema.europa. eu/en/human-regulatory/marketing-authorisation.

10. Hollak CE, Aerts JM, Aymé S, Manuel J. Limitations of drug registries to evaluate orphan medicinal products for the treatment of lysosomal storage disorders. Orphanet J Rare Dis. 2011;6:16.

11. Germain DP, Elliott PM, Falissard B, Fomin W, Hilz MJ, Jovanovic A, et al. The effect of enzyme replacement therapy on clinical outcomes in male patients with Fabry disease: a systematic literature review by a European panel of experts. Mol Genet Metab. 2019;19:100454.

12. Stapleton M, Hoshina H, Sawamoto K, Kubaski F, Mason RW, Mackenzie WG, et al. Critical review of current MPS guidelines and management. Mol Genet Metab. 2019;126(3):238-45

13. Download medicine data [internet]. Amsterdam: European Medicines Agency; c1995-2019 [cited 2019 Dec 31]. Available from: https://www.ema. europa.eu/sites/default/files/Medicines_output_european_public_ assessment_reports.xIsx.

14. EU PAS Register [internet]. Amsterdam: European Medicines Agency; c19952016 [cited 2019 Dec 31 through 2020 Jan 2]. Available from: http://www. encepp.eu/encepp_studies/indexRegister.shtml.

15. Medicines [internet]. Amsterdam: European Medicines Agency; c1995-2020 [cited 2019 Dec 31 through 2020 Jan 2]. Available from: https://www.ema. europa.eu/en/medicines.

16. Meikle PJ, Fietz MJ, Hopwood JJ. Diagnosis of lysosomal storage disorders: current techniques and future directions. Expert Rev Mol Diagn. 2004;4(5): 677-91

17. Beutler E. The cost of treating Gaucher disease. Nat Med. 1996:2(5):523-4.

18. McCabe ERB, Fine BA, Golbus MS, Greenhouse JB, McGrath GL, New M, et al Gaucher disease. Current issues in diagnosis and treatment. NIH technology assessment panel on Gaucher disease. JAMA. 1996;275(7):548-53. 
19. Mistry PK, Batista JL, Andersson HC, Balwani M, Burrow TA, Charrow J, et al. Transformation in pretreatment manifestations of Gaucher disease type 1 during two decades of alglucerase/imiglucerase enzyme replacement therapy in the international collaborative Gaucher group (ICGG) Gaucher registry. Am J Hematol. 2017;92(9):929-39.

20. Belmatoug N, Di Rocco M, Fraga C, Giraldo P, Hughes D, Lukina E, et al. Management and monitoring recommendations for the use of eliglustat in adults with type 1 Gaucher disease in Europe. Eur J Intern Med. 2017;37:25-32.

21. Wasserstein MP, Caggana M, Bailey SM, Desnick RJ, Edelmann L, Estrella L, et al. The New York pilot newborn screening program for lysosomal storage diseases: report of the first 65,000 infants. Genet Med. 2019;21(3):631-40.

22. El Dib R, Gomaa H, Ortiz A, Politei J, Kapoor A, Barreto F. Enzyme replacement therapy for Anderson-Fabry disease: a complementary overview of a Cochrane publication through a linear regression and a pooled analysis of proportions from cohort studies. PLoS One. 2017:12(3):1-22.

23. Sirrs $S$, Clarke JTR, Bichet DG, Casey R, Lemoine K, Flowerdew G, et al. Baseline characteristics of patients enrolled in the Canadian Fabry disease initiative. Mol Genet Metab. 2010;99:367-73.

24. Biegstraaten M, Arngrímsson R, Barbey F, Boks L, Cecchi F, Deegan PB, et al. Recommendations for initiation and cessation of enzyme replacement therapy in patients with Fabry disease: the European Fabry working group consensus document. Orphanet J Rare Dis. 2015;10:36.

25. Burton BK, Silliman N, Marulkar S. Progression of liver disease in children and adults with lysosomal acid lipase deficiency. Curr Med Res Opin. 2017; 33(7):1211-4.

26. Bellgard MI, Napier KR, Bittles AH, Szer J, Fletcher S, Zeps N, et al. Design of a framework for the deployment of collaborative independent rare diseasecentric registries: Gaucher disease registry model. Blood Cells Mol Dis. 2018; 68:232-8.

27. Moynihan R, Bero L, Hill S, Johansson M, Lexchin J, MacDonald H, et al. Pathways to independence: towards producing and using trustworthy evidence. BMJ. 2019;367:16576 1-5.

28. Moseley J, Vamvakas S, Berntgen M, Cave A, Kurz X, Arlett P, et al. Regulatory and health technology assessment advice on postlicensing and postlaunch evidence generation is a foundation for lifecycle data collection for medicines. Br J Clin Pharmacol. 2020;86(6):1034-51.

29. Koch C, Schleeff J, Techen F, Wollschläger D, Schott G, Kölbel R, et al. Impact of physicians' participation in non-interventional post-marketing studies on their prescription habits: a retrospective 2-armed cohort study in Germany. PLoS Med. 2020;17(6):e1003151.

\section{Publisher's Note}

Springer Nature remains neutral with regard to jurisdictional claims in published maps and institutional affiliations.

Ready to submit your research? Choose BMC and benefit from:

- fast, convenient online submission

- thorough peer review by experienced researchers in your field

- rapid publication on acceptance

- support for research data, including large and complex data types

- gold Open Access which fosters wider collaboration and increased citations

- maximum visibility for your research: over $100 \mathrm{M}$ website views per year

At $\mathrm{BMC}$, research is always in progress.

Learn more biomedcentral.com/submissions 